\title{
The Pathology of Amyloidosis in Classification: A Review
}

\author{
Maria M. Picken \\ Department of Pathology, Loyola University Medical Center, Maywood, IL, USA
}

\section{Keywords}

Amyloidosis · Hereditary · Organ system · Pathology

\begin{abstract}
Background: The amyloidoses are a rare and heterogeneous group of disorders that are characterized by the deposition of abnormally folded proteins in tissues ultimately leading to organ damage. The deposits are mainly extracellular and are recognizable by their affinity for Congo red and their yellow-green birefringence under polarized light. Current classification of amyloid in medical practice is based on the amyloid protein type. To date, 36 proteins have been identified as being amyloidogenic in humans. Summary: in clinical practice, it is critical to distinguish between treatable versus non-treatable amyloidoses. Moreover, amyloidoses with a genetic component must be distinguished from the sporadic types and systemic amyloidoses must be distinguished from the localized forms. Among the systemic amyloidoses, $\mathrm{AL}$ continues to be the most common amyloid diagnosis in the developed world; other clinically significant types include AA, ALECT2, and ATTR. The latter is emerging as an underdiagnosed type in both the hereditary and wild-type setting. Other hereditary amyloidoses include AFib, several amyloidoses derived from apolipoproteins, AGel, ALys, etc. In a dialysis setting, systemic amyloid derived from $\beta_{2}$ microglobulin $(A \beta 2 M)$ should be considered, although a very rare hereditary variant has also been reported; several amyloido-
\end{abstract}

ses may be typically associated with aging and several iatrogenic types have also emerged. Determination of the amyloid protein type is imperative before specific therapy can be implemented and the current methods are briefly summarized. A brief overview of the target organ involvement by amyloid type is also included. Key Messages: (1) Early diagnosis of amyloidosis continues to pose a significant challenge and requires the participation of many clinical and laboratory specialties. (2) Determination of the protein type is imperative before specific therapy can be implemented. (3) While mass spectrometry has emerged as the preferred method of amyloid typing, careful application of immune methods is still clinically useful but caution and experience, as well as awareness of the limitations of each method, are necessary in their interpretation. (4) While the spectrum of amyloidoses continues to expand, it is critical to distinguish between those that are currently treatable versus those that are untreatable and avoid causing harm by inappropriate treatment.

(c) 2020 S. Karger AG, Basel

\section{Introduction}

The amyloidoses are a rare and heterogeneous group of disorders that are characterized by the deposition of abnormally folded proteins in tissues. Amyloid deposits are formed from globular, soluble proteins, which under- karger@karger.com

(c) 2020 S. Karger AG, Basel

Karger!
Dr. Maria M. Picken

Department of Pathology, Loyola University Medical Center 2160 S First Ave

Maywood, IL 60153 (USA)

MPICKEN@lumc.edu 
go misfolding and, subsequently, aggregate into insoluble fibrils, leading to progressive organ damage. By electron microscopy, amyloid fibrils appear rigid, are non-branching, and typically measure 8-12 $\mathrm{nm}$ in diameter [1].

Fibrillogenesis involves abnormal protein folding leading to a conformational shift into a $\beta$-pleated sheet secondary structure consequent to which the protein becomes hydrophobic, insoluble, non-functional, and resistant to degradation. Amyloid formation involves a combination of several factors, including: a sustained increase in the concentration of proteins with an acquired or hereditary mutation, or wild-type proteins with an intrinsic propensity to misfold, or a proteolytic remodeling of a wild-type protein into an amyloidogenic fragment [2-4].

Protein folding is under the control of quality control systems, which operate at the cellular (as well as extracellular) level and promptly eliminate misfolded proteins. It is only when these quality control systems are overwhelmed by the conditions listed above, or are reduced in their capacity by aging, that amyloidogenesis occurs. In this process, misfolded proteins are generated and aggregate as protofibrils and ultimately mature fibrils. Importantly, amyloid toxicity (referred to as proteotoxicity) begins at the level of the protofibril and is continued by the mature fibril. Mature fibrils have binding sites for Congo red. During fibrillogenesis, amyloid P component, apoliporotein $\mathrm{E}$, and glycosaminoglycans contribute to the formation and persistence of amyloid deposits. These components are found in all amyloid deposits, regardless of the protein type, and therefore serve as universal amyloid signatures [2-4].

This review will focus on the extracerebral amyloidoses and the practical issues associated with their diagnosis and classification. First, the rationale for the modern classification of amyloidoses will be discussed followed by a brief overview of the major amyloidoses, focusing on systemic types, diagnosis, and, finally, an organ systembased summary.

\section{Classification}

As per the International Society of Amyloidosis (ISA) guidelines, in human medicine, the term amyloid is applied to mainly extracellular deposits of a fibrillary protein that are recognizable by their affinity for Congo red and their yellow-green birefringence under polarized light [1].

The current classification of amyloid in medical practice is based on the amyloid protein type. The amyloid is termed A (for amyloid) followed by an abbreviation of the protein type: AL (amyloid derived from immunoglobulin light chain), ATTR (amyloid derived from transthyretin), etc. Table 1 provides an abbreviated list of amyloid fibril proteins and their precursors in humans. Amyloid protein variants associated with hereditary amyloidoses are named according to the substitution or deletion in the mature protein; thus, the name of the amino acid involved and the position of the change are listed: e.g., ATTRV30M (valine is replaced by methionine). While the single-letter amino acid code is recommended by the ISA, frequently, the three-letter amino acid designation is used in the literature, in keeping with the recommendations of the Sequence Variant Description Working Group of the Human Genome Variation Society. The name "hereditary" rather than "familial" is recommended by the ISA for amyloid diseases associated with mutant proteins. Moreover, "hereditary ATTRv," where "v" stands for variant instead of " $\mathrm{m}$ " for mutant, is recommended by the ISA; however, at times, ATTRm (or hATTR for hereditary ATTR) may be encountered in the literature as an alternative to ATTRv. The designation ATTRwt is frequently used to underscore the association with wild-type protein in contrast to the variant.

To date, 36 proteins have been identified as being amyloidogenic in humans. Amyloid deposits may be localized or systemic, with deposits being present in a single organ in the former case and affecting various organs and tissues throughout the body in the latter. Exclusively localized amyloid deposits have been associated with at least 19 protein types, while at least 14 protein types (and many more variants) appear to be consistently associated with systemic amyloidosis. Interestingly, however, at least 3 protein types (most notably AL/AH, amyloidosis derived from immunoglobulin light or heavy chain, respectively, ATTR, and amyloidosis derived from $\beta_{2}$ microglobulin, $A \beta 2 \mathrm{M}$ ) can occur as either localized or systemic deposits.

Clinical manifestations are heterogeneous and may be influenced by genetic and environmental factors. Although certain phenotypes may typically be associated with certain amyloid types, considerable clinical overlap exists and, hence, the classification of amyloidoses on clinical grounds alone is not recommended.

In clinical practice, it is critical to distinguish between treatable versus non-treatable amyloidoses. Moreover, amyloidoses with a genetic component must be distinguished from the sporadic types and systemic amyloidoses must be distinguished from the localized forms [5$12]$. 
Table 1. Classification of amyloidoses

\begin{tabular}{|c|c|c|c|c|}
\hline Fibril protein & Precursor protein & $\begin{array}{l}\text { Systemic (S), } \\
\text { localized (L) }\end{array}$ & $\begin{array}{l}\text { Acquired (A), } \\
\text { hereditary }(\mathrm{H})\end{array}$ & Target organs \\
\hline $\mathrm{AL}, \mathrm{AH}$ & $\begin{array}{l}\text { Immunoglobulin } \\
\text { Light }(\mathrm{L}) \text { or heavy }(\mathrm{H}) \text { chain }\end{array}$ & $\mathrm{S}, \mathrm{L}$ & $\mathrm{A}(\mathrm{H})^{*}$ & $\begin{array}{l}\text { All organs, usually except } \\
\text { CNS** }\end{array}$ \\
\hline AA & (Apo) serum amyloid A & S & $\mathrm{A}$ & All organs, except $\mathrm{PNS}^{\#}, \mathrm{CNS}$ \\
\hline ALECT2 & Leukocyte chemotactic factor- 2 & $\mathrm{~S}$ & A & $\begin{array}{l}\text { Kidney primarily } \\
\text { liver }\end{array}$ \\
\hline ATTRv & Transthyretin variants (v) & $\mathrm{S}$ & $\mathrm{H}$ & $\begin{array}{l}\text { Heart, PNS, ANS \#\#, eye } \\
\text { leptomeninges }\end{array}$ \\
\hline AFib & Fibrinogen A $\alpha$ chain variants & $\mathrm{S}$ & $\mathrm{H}$ & Kidney primarily \\
\hline $\begin{array}{l}\text { Apolipoproteins } \\
\text { AI, AII, CII, CIII }\end{array}$ & Apolipoprotein variants & S & $\mathrm{H}$ & $\begin{array}{l}\text { all variants kidney, } \\
\text { AApoAI also heart, liver, PNS, } \\
\text { testis, larynx, skin }\end{array}$ \\
\hline $\mathrm{A} \beta 2 \mathrm{Mv}$ & $\beta_{2}$ microglobulin variant & S & $\mathrm{H}$ & ANS \\
\hline ATTRwt & Wild type, aging & S & $\mathrm{A}$ & $\begin{array}{l}\text { Cardiac primarily, lung, } \\
\text { ligaments, tenosynovium }\end{array}$ \\
\hline AApoAIV & $\begin{array}{l}\text { Apolipoprotein AIV } \\
\text { Wild type, aging }\end{array}$ & $\mathrm{S}$ & $\mathrm{A}$ & Kidney medulla, systemic \\
\hline ASem 1 & $\begin{array}{l}\text { Semenogelin } 1 \\
\text { Wild type, aging }\end{array}$ & $\mathrm{L}$ & $\mathrm{A}$ & Seminal vesicles \\
\hline $\begin{array}{l}\text { A } \beta 2 \mathrm{M} \\
\text { AIns (insulin) } \\
\text { AEnf (enfurvitide) }\end{array}$ & $\begin{array}{l}\text { Wild type, iatrogenic (dialysis) } \\
\text { Iatrogenic } \\
\text { Iatrogenic }\end{array}$ & $\begin{array}{l}\mathrm{S} \\
\mathrm{L} \\
\mathrm{L}\end{array}$ & $\begin{array}{l}\text { A } \\
\text { A } \\
\text { A }\end{array}$ & $\begin{array}{l}\text { Musculo-skeletal system } \\
\text { Injection sites }\end{array}$ \\
\hline
\end{tabular}

* Extremely rare cases of hereditary AL have also been included [1]. ${ }^{* *}$ Central nervous system. ${ }^{\#}$ Peripheral nervous system. ${ }^{\#}$ Autonomic nervous system. ${ }^{\wedge}$ Atrial natriuretic factor. ${ }^{\wedge}$ Islet amyloid polypeptide.

\section{Main Amyloidoses}

$\mathrm{AL}$, amyloidosis derived from immunoglobulin light chain, has been considered, thus far, the most common form of systemic amyloidosis in the developed world [6, $7,12,13]$.

Approximately 2,200 new cases of AL are diagnosed every year in the US. The prevalence of AL amyloidosis has increased significantly (2.6-fold) between 2007 and 2015, from 15.5 cases per million in 2007 to 40.5 in 2015 [13]. Since AL is discussed in several chapters in this issue, here, it will be addressed only briefly in the context of other amyloidoses.

By definition, AL is associated with the clonal proliferation of plasma cells, producing a monoclonal protein that circulates in the blood and amyloid fibrils are derived from the immunoglobulin light chain or its fragment; rare cases that are derived from a heavy chain fragment are designated AH. Only $20 \%$ of AL amyloidosis patients present with an accompanying multiple myeloma; the majority are diagnosed with plasma cell dyscrasia. Importantly, AL amyloidosis may be associated with near nor- 


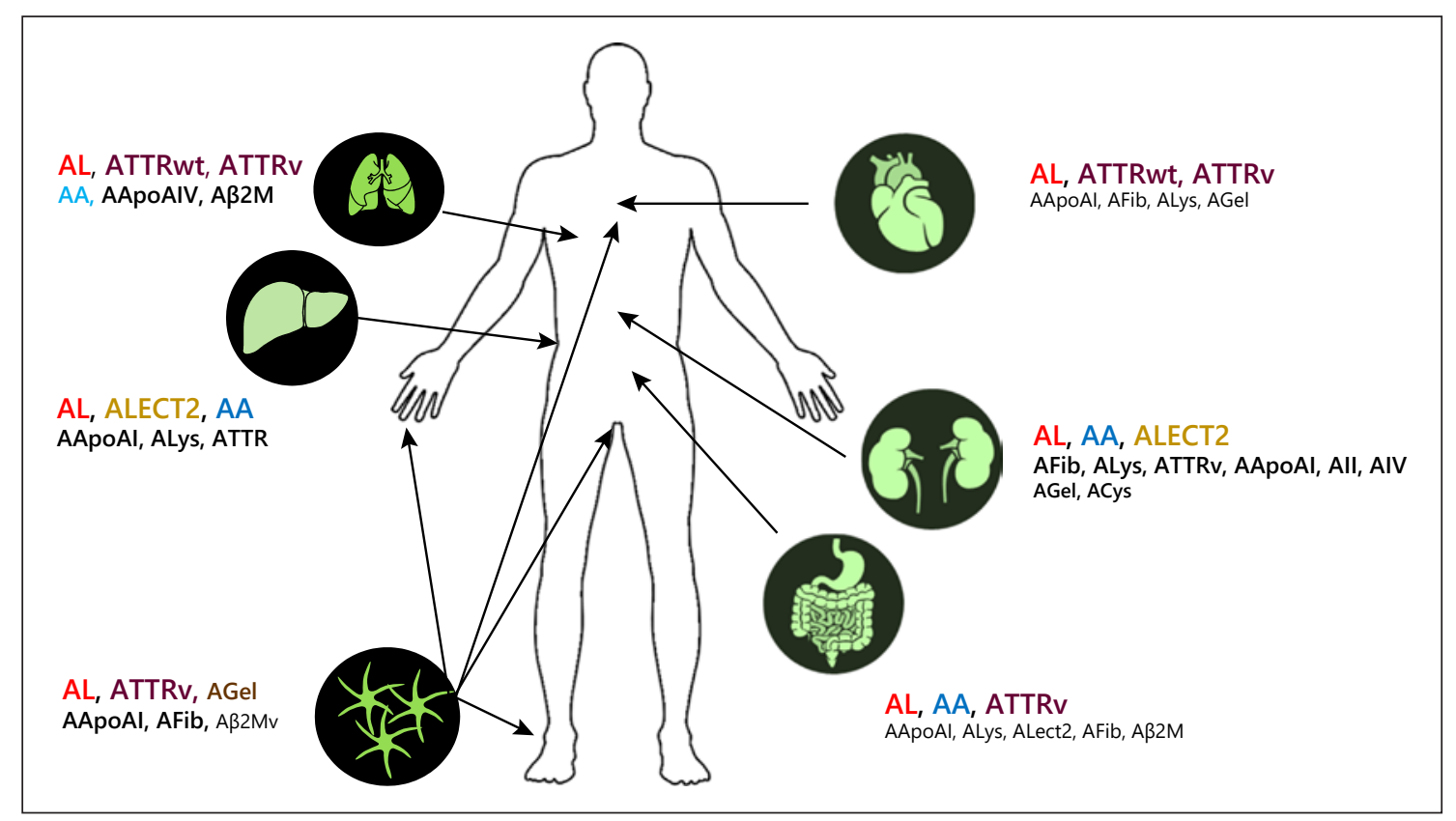

Fig. 1. Systemic amyloidosis types by target organ involvement.

mal counts and polyclonal plasma cells in a bone marrow biopsy $[6,7,12,13]$. Extremely rare cases of hereditary AL have also been included [1].

Approximately $70 \%$ of patients present with renal involvement - typically with nephrotic syndrome - and an equal proportion of patients present with cardiac involvement - heart failure and arrhythmias [6, 7, 12-14]. Between $17-35 \%$ of patients with AL have clinically significant neuropathy. Importantly, the AL phenotype, with cardiomyopathy and/or peripheral neuropathy, overlaps with hereditary amyloidoses (Fig. 1).

\section{AA}

In AA amyloidosis, amyloid fibrils are derived from serum amyloid A protein, which is an acute phase reactant produced by the liver $[15,16]$. AA is typically associated with an underlying chronic inflammatory process and remains the leading cause of systemic amyloidosis in developing countries, due to the high prevalence of associated, underlying, infectious diseases [15, 17-19]. AA can develop in association with hereditary auto-inflammatory diseases, both monogenic (familial Mediterranean fever) as well as polygenic, including inflammatory bowel disease [15]. It is important to recognize the distinction between the familial amyloidoses (i.e., familial AA associat- ed with autoinflammatory diseases) versus those referred to as "hereditary" amyloidoses. In the former, the genetic abnormality affects the protein(s) involved in modulation of the inflammatory response, which, in turn, creates an environment that is permissive for AA development [15]. In contrast, in hereditary amyloidoses, the mutation involves the amyloidogenic protein itself and, thereby, makes it prone to amyloidogenesis. The list of conditions associated with AA continues to expand and, most recently, obesity was added as a significant susceptibility factor for idiopathic AA [20]. It is, therefore, important to monitor patients at risk. However, no association with an identifiable underlying disease can be seen in up to $19 \%$ of patients diagnosed with AA $[15,17]$. Interestingly, while a chronic, sustained inflammatory process may lead to the development of AA amyloidosis, not every patient with chronic disease develops AA. The typical phenotype of AA amyloidosis includes involvement of the kidneys, gastrointestinal tract, spleen, and liver [15, 18] (Fig. 1).

\section{ALECT2}

ALECT2 accounts for $2.7-10 \%$ of patients with renal amyloidosis in the United States; thus, overall, it represents the third most common renal amyloidosis after $\mathrm{AL}$ and AA [21]. However, the proportion of patients affect- 
ed depends on ethnicity $[19,21,22]$. Remarkably, in the Southwestern United States, which has a high proportion of Mexican Americans, ALECT2 accounts for 54\% of amyloid diagnoses and has emerged as the most prevalent renal amyloidosis in Mexican Americans [21]. In Egypt, ALECT2 is the second most common renal amyloidosis type after AA [19].

In ALECT2, the amyloid fibrils are derived from leukocyte chemotactic factor 2 [23]. However, the pathogenesis is unclear: no genetic component has emerged as yet, despite a strong association with Mexican American ethnicity. Additional ethnic groups that are prone to develop ALECT2 include: Native Americans, First Nations people in British Columbia, Punjabis, and Egyptians [19, 21-27].

ALECT2 affects the older patient population, typically presents with slowly progressive renal failure, and is an important cause of end-stage renal disease [25-27]. While ALECT2 is a systemic amyloidosis with predominant involvement of the kidney and liver, subclinical involvement has been reported in the spleen, lungs, prostate, gallbladder, pancreas, small bowel, and adrenal and parathyroid gland; however, to date, no patient with cardiac ALECT2 amyloidosis has been confirmed histologically [22-27] (Fig. 1).

\section{Hereditary Amyloidoses}

Among the hereditary amyloidoses, most types are individually rare but, collectively, hereditary amyloidoses constitute approximately $10 \%$ of all systemic amyloidoses currently diagnosed [28]. This may, however, represent underdiagnosis. ATTRv is the most common hereditary amyloidosis worldwide. AFib (amyloidosis derived from a fibrinogen variant) is rare in North America but more common in Europe; other rarer examples of hereditary amyloidoses include diseases associated with variants of apolipoproteins (AI, AII, C-II, C-III), gelsolin (AGel), and lysozyme (ALys) [5, 12, 28-33].

Hereditary amyloidosis caused by mutations in the TTR gene (TTRv, variant) has begun to emerge as a significantly underdiagnosed cause of cardiac failure and polyneuropathy $[8-10,12]$. The worldwide prevalence of ATTRv is estimated at 50,000 individuals, with varying phenotypic presentations [29].

Transthyretin, a protein primarily produced by the liver, comprises four monomers forming a tetramer in the mature protein and is involved in the transport of thyroxine and retinol. TTR gene mutations can lead to weaker monomer interactions, leading, in turn, to dissociation of the tetramer. Monomers can misfold and then aggregate into amyloid fibrils [12].

In the United States, the most common transthyretin mutation is Val122Ile, which affects 3-4\% of African Americans. In Sweden, Portugal, and Japan, there are endemic foci of Val30Met [34, 12]. To date, there are more than 120 TTR mutations reported. Val122Ile is predominantly cardiopathic, while Val30Met is predominantly associated with polyneuropathy; the prevalence of other rarer mutations varies according to geographical location and these mutations appear to be associated with more pronounced cardiomyopathy rather than polyneuropathy [28].

The majority of patients are male heterozygous carriers. Hereditary TTR amyloidosis is autosomal dominant with variable penetrance and variable age at onset; hence, a family history is often missing. The median age at onset can vary depending on geographic location: in the US 68 years, in Portugal - 32 years, in Sweden - 52 years [9, $12,35]$. But even in similar geographic locations, the age range of patients can be fairly wide. Amyloid deposits typically contain a mixture of both mutant and wild-type TTR. Moreover, in Val30Met, fibrils may be composed of either the full-length TTR molecule or a mixture of fulllength TTR and fragments thereof and the fibril composition may be associated with the age at onset of the disease and the degree of cardiac involvement [35].

Phenotypically, there is a considerable overlap between AL and ATTRv amyloidosis with both being associated with cardiomyopathy and peripheral neuropathy [9, 10, 12] (Fig. 1). Moreover, a substantial proportion of patients with ATTR also have MGUS - in a recent study, $49 \%$ of patients with cardiac ATTRv amyloidosis were shown to have a concurrent MGUS [36]. Regrettably, some ATTRv patients have been misdiagnosed as having AL amyloidosis.

AFib - amyloidosis derived from a fibrinogen variant - has a worldwide distribution [32, 33, 37-39]. It is the most frequent hereditary amyloidosis in Northern Europe but is rare in the US $(1 \%)[21,32,33]$. Fibrinogen, a plasma protein involved in the final phase of blood coagulation, is produced exclusively by the liver and the amyloidogenic mutation involves the fibrinogen A $\alpha$-chain. Several variants have been reported that usually have no effect on fibrinogen function, with the exception of deletions associated with frame shift mutations. Some patients have decreased fibrinogen levels. Median age at presentation is 55 years. The typical presentation involves nephrotic syndrome and hypertension. Pathology usually shows massive glomerular amyloid with essentially no extraglomerular deposits. Spleen involvement may be asso- 
ciated with anemia and spleen rupture. A family history is frequently missing. Certain mutations may be associated with neuropathy [28].

Other hereditary amyloidoses include several associated with variants of apolipoproteins (AI, AII, C-II, CIII), gelsolin (AGel), and lysozyme (ALys) [28].

AApoAI (amyloidosis derived from an apolipoprotein AI variant) is the most common type encountered in this group. Mutations in the aminoterminal portion of the protein are associated with renal and/or hepatic amyloid deposits. The former are typically extraglomerular with dense deposits at the cortico-medullary junction and are associated with renal failure. Certain mutations are associated with hepatic, testicular, laryngeal, and cutaneous deposits as well as peripheral neuropathy and/or cardiomyopathy. AApoAII is primarily nephropathic [28].

Amyloidosis derived from a gelsolin variant, AGel, is much less common than ATTRv and is associated with predominant seventh cranial nerve neuropathy, early and prominent corneal lattice dystrophy, cutis laxa, and, later, in the course of the disease, a distal polyneuropathy. AGel follows a benign course with relatively late onset, slow progression, and limited morbidity. Most known patients have been reported in Finland, but patients from the United States, Denmark, the Netherlands, and Japan have also been encountered [28].

ALys - amyloid derived from lysozyme - is particularly nephropathic; massive hepatic deposits may lead to hepatic rupture. Cardiomyopathy and gastrointestinal involvement have also been reported.

Hereditary and systemic amyloidosis derived from a $\beta_{2}$ microglobulin variant $(\mathrm{A} \beta 2 \mathrm{M})$ is exceedingly rare [40]. It was reported with autonomic and subsequent symmetric sensorimotor axonal polyneuropathy. This is in contrast to dialysis-associated amyloidosis, derived from wildtype $\beta_{2}$ microglobulin, where there is no associated polyneuropathy.

\section{Amyloidoses of Aging}

Several amyloidoses are associated with aging, including systemic (ATTRwt, AApoAIV) and localized forms (atrial and seminal vesicle amyloid) [41-47]. Development of these amyloidoses may be associated with agerelated reduced effectiveness of the protein folding quality control systems.

ATTR, derived from the wild-type protein (ATTRwt) and causing predominantly cardiac failure, is recognized as an underdiagnosed cause of cardiac failure in older pa- tients, predominantly males. ATTRwt, formerly called "senile systemic amyloidosis," is increasing rapidly in recognition and, with an aging population, ATTRwt is likely to become the most common type of cardiac amyloidosis $[9,12]$.

With aging, the TTR tetramer becomes less stable, resulting in the release of misfolded intermediates that ultimately form amyloid deposits, mainly in the heart. Most commonly, ATTRwt is reported in men over the age of 75 years. Autopsy data have shown TTR deposits in the myocardium of $\approx 25 \%$ of individuals $>80$ years old $[9,42]$. While extracardiac amyloid deposits are largely limited to the vasculature, amyloid associated with carpal tunnel syndrome and lumbar spine stenosis may also be detected [43-46].

A substantial proportion of patients with ATTR also have MGUS. In a recent study, 39\% of patients with cardiac, wild-type, ATTR amyloidosis had concurrent MGUS and, hence, there is a danger of misdiagnosis [36].

AApoAIV predominantly involves the renal medulla but systemic deposits in the small intestine, heart, and, very rarely, in the lung and skin were detected by mass spectrometry studies.

Atrial localized amyloid, derived from atrial natriuretic factor (a natriuretic peptide hormone secreted from the cardiac atria), may be associated with atrial fibrillation that is refractory to medical treatment [47].

Localized amyloid deposits, which may involve cardiac valves and the aorta, are considered to represent lo$\mathrm{cal}$, age-related amyloidosis and, in most instances, are asymptomatic and incidental findings [46].

Localized amyloid involving seminal vesicles in elderly men is derived from semenogelin 1 [46].

\section{latrogenic Amyloidoses}

$\mathrm{A} \beta 2 \mathrm{M}$, amyloidosis derived from $\beta_{2}$ microglobulin, is associated with long-term dialysis [1]. Also, localized deposits of amyloid derived from insulin and enfurvitide have been associated with the injection sites of these drugs in the case of long-term treatment $[1,46]$.

\section{Localized Amyloid Deposits}

Exclusively localized amyloid deposits may be associated with several endocrine organs or tumors, where they are derived from the respective hormones or local protein precursors $[1,46]$. 

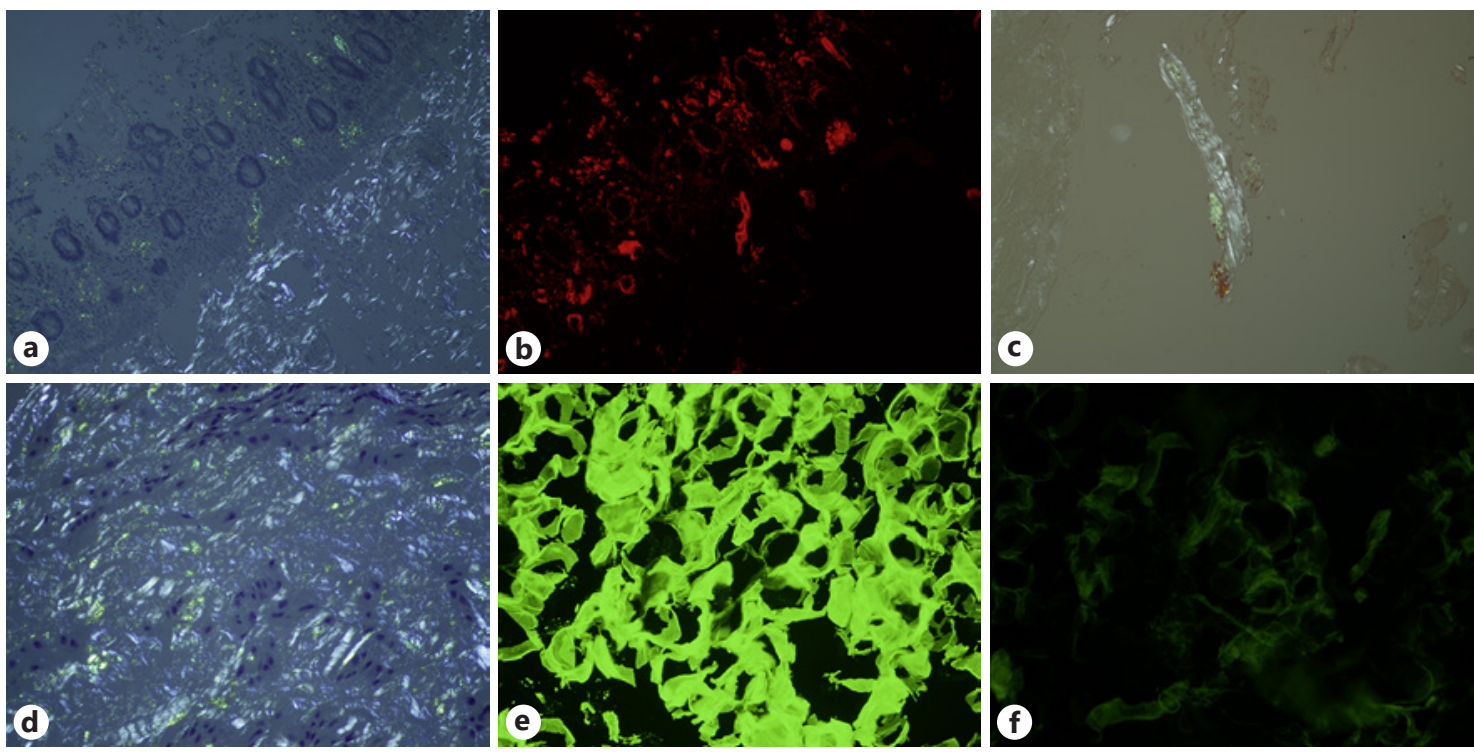

Fig. 2. a Colon biopsy positive for amyloid by Congo red stain. Small deposits of amyloid are seen in the lamina propria, focally in muscularis mucosae and vasculature. Congo red stain viewed under polarized light. Original magnification $100 \times$. b Colon biopsy, the same field as in a. Amyloid deposits are more readily visible. Congo red stain viewed under fluorescence light using TRITC filter. Original magnification $100 \times$. c Bone marrow biopsy specimen with periosseous soft tissue positive for amyloid while the actual

Localized deposits of amyloid may also involve the pulmonary and lower urinary tracts [46]. While these deposits may be multifocal and bilateral, they involve a single organ system. Deposits of amyloid are most frequently of the AL type, derived from mucosa-associated lymphoid tissue (MALT) lymphoma [48]. Localized deposits of amyloid may form a tumor-like mass, referred to as an "amyloidoma," which may be seen in peripheral nerves and, rarely, in the gastrointestinal tract.

\section{Diagnosis}

Amyloidosis should be suspected in patients with nondiabetic nephrotic proteinuria, heart failure with preserved ejection fraction, non-diabetic neuropathy, or unexplained gastrointestinal symptoms/hepatomegaly [57]. However, except for patients with a positive pyrophosphate scan (and absence of paraprotein), a diagnosis of amyloidosis is based on the tissue detection of deposits $[29,49,50]$.

Congo red stain under polarized light is the current gold standard for a "generic" diagnosis of amyloidosis i.e., regardless of the protein type. A subsequent step in- marrow was negative (not shown). Congo red stain. Original magnification 200x. d Carpal tunnel biopsy specimen positive for amyloid by Congo red stain while amyloid deposits were inconspicuous by H\&E stain (not shown). Original magnification $200 \times$. e, f Fat biopsy stained with anti-lambda light chain antibody (e) and anti-kappa light chain antibody (f) illustrating lambda light chain restriction. Immunofluorescence stains in frozen section. Original magnification $400 \times$.

volves the identification of the specific amyloid protein type - "amyloid typing" - which, in turn, has direct implications for the choice of therapy $[1,50]$.

While, in the routine pathology stain H\&E, amyloid has a nonspecific appearance as extracellular "amorphous" deposits, early deposits may be inconspicuous. Hence, a Congo red stain should be examined to rule out early amyloid and not just to confirm a suspicion of amyloid based on H\&E. The sensitivity of the Congo red stain may be greatly enhanced by combining it with fluorescence microscopy [50, 51] (Fig. 2a, b). In addition to Congo red stain, Thioflavin may merit consideration [52]. Pathology reporting of tissue positivity for amyloid should specify the location of deposits (stromal versus vascular), since it is relevant clinically for the classification of organ involvement [14].

While biopsy of a target organ (kidney, heart) is most sensitive if amyloid is clinically suspected, a less invasive biopsy is recommended, such as abdominal fat biopsy and bone marrow biopsy [50-56]. The sensitivity of fat in amyloid detection is amyloid-type dependent, ranging from $70-90 \%$ in AL and $67 \%$ in ATTRv to only $14 \%$ in ATTRwt [53]. Fine needle fat biopsy typically yields a small amount of tissue; hence, a small excisional biopsy 
has been advocated, which increases the likelihood of amyloid detection and provides ample tissue for amyloid typing $[50,56,57]$.

Bone marrow biopsy is routinely performed for the diagnosis/follow-up of patients with most hematologic disorders. The reported overall sensitivity of this procedure for amyloid detection is $\sim 60 \%$ and may be the basis of a first-time diagnosis of amyloidosis in up to one-third of patients $[6,51,58,59]$. In bone marrow biopsies selected for Congo red stain because of light chain restriction, increased plasma cells, MGUS, clinical suspicion, or prior diagnosis of amyloidosis in other tissues, amyloid was detected in 10.5\% [51]. Bone marrow biopsy findings in non-AL patients, may also be useful since there is evidence that at least AA, ATTR, and ALECT2 may be associated with vascular and/or extramedullary deposits of amyloid $[21,26,51,60]$. The sensitivity of bone marrow biopsy in ATTRv has been reported as $41 \%$, while in ATTRwt it is reported as 30\% [53].

In patients with $\mathrm{AL}$, both samples (fat and bone marrow biopsy) were negative for amyloid in only $14.6 \%$ [6]. Therefore, bone marrow biopsy, together with fat pad biopsy, may obviate the need for more invasive target organ biopsies in a significant number of patients [51, 61].

In bone marrow biopsy specimens, amyloid deposits may be seen in the vessel wall, bone marrow stroma, periosteal soft tissue, or a combination thereof. Importantly, non-stromal deposits are more than 2.25 times more common than stromal deposits. Hence, it is important to look for amyloid in periosteal soft tissue in bone marrow biopsy specimens [51] (Fig. 2c).

While labial and rectal biopsies have been used extensively for screening in the past, more recently, luminal gastro-intestinal biopsies have been utilized as well. In AL amyloidosis, labial salivary gland biopsies have reported $81-89 \%$ sensitivity, while gastrointestinal biopsies have $70-90 \%$ sensitivity. In ATTRv, rectal biopsy sensitivity is $81 \%$, while in ATTRwt, it is 50\% $[53,56]$. Moreover, testing for amyloid in tenosynovial and carpal tunnel specimens has also been advocated [43-46] (Fig. 2d).

\section{Amyloid Typing}

Determination of the amyloid protein type is imperative before specific therapy can be implemented [5-12] There is significant heterogeneity in the spatial distribution of amyloid in various tissues and, while there is some predilection of certain amyloid types for particular organs, the determination of the amyloid type on clinical grounds alone is not reliable; the amyloid protein type must be determined by immune and/or proteomic examination of the actual amyloid deposits. Recently, PYP scans have been advocated for the radiologic detection of ATTR cardiomyopathy in patients without MGUS [49].

In the US and other developed countries, the main differential diagnosis of the amyloid protein type is between AL and non-AL types. Among clinical amyloidoses diagnosed at the Mayo clinic by proteomics (regardless of the specimen site), AL was the most frequent diagnosis (61.7\%), followed by ATTR (24.5\%), with other types being much rarer - AA (3.7\%) and ALECT2 (3.6\%) [21].

While immunofluorescence in frozen sections and immunohistochemistry in paraffin sections have traditionally been used for amyloid typing, mass spectrometry has emerged as the method of choice for the typing of amyloid proteins, in particular in paraffin sections $[6-12,62-$ 72].

In general, immunofluorescence in frozen sections performs better than immunohistochemistry in paraffin sections and continues to be used as a first step in amyloid typing by many laboratories worldwide [21, 62] (Fig. 2e, f). More recently, the application of paraffin-section immunofluorescence has been reported as superior to routine paraffin-section immunohistochemistry, in particular for the detection of AL [68-70]. Moreover, some centers continue to use immunogold labelling with good results and the application of antibodies raised against free light chains (as opposed to conventional antibodies) may also yield better results $[73,74]$. While detailed discussion of the issues associated with amyloid typing by immunohistochemistry and mass spectrometry are beyond the scope of this review, suffice it to say that caution and experience, as well as awareness of the limitations of each method, are necessary in their interpretation.

The rationale for the application of proteomics to amyloid typing lies in the relative abundance of amyloid protein in the tissue, where, usually, amyloid protein is the dominant protein [64-66]. Thus, the diagnosis of amyloid by proteomic methods is based on the presence of large spectra numbers for the amyloidogenic protein in conjunction with apolipoprotein $\mathrm{E}$ and serum amyloid $\mathrm{P}$ component (also known as "amyloid signature"). Importantly, mass spectrometry proteomics allows global identification of proteins and, thereby, the discovery of unsuspected amyloid protein types.

Currently, amyloid typing by mass spectrometry is recommended for the typing of amyloid deposits where routine IF/IHC is negative or equivocal, for the detection of less common/unusual types, for the confirmation of an 
unusual or unexpected amyloid type, or in cases with an inadequate sample for immunofluorescence typing [21].

Amyloid protein typing must be done on the actual tissue deposits. Ancillary laboratory studies are used to support the diagnosis of the amyloid protein type but not to make it! Thus, the detection of MGUS is not in itself diagnostic of AL and caution is mandatory since as many as $10-49 \%$ of patients with ATTR may have MGUS [36]. In cases of hereditary amyloidosis, gene sequencing is recommended since hereditary amyloidosis types cannot be distinguished from acquired types based on clinical presentation alone.

Amyloidosis staging involves the distinction between systemic and localized amyloidoses as well as determination of the organ type involvement. These issues are discussed by Schonland et al. elsewhere in this issue.

\section{Target Organ - Amyloid Involvement by Type}

\section{Kidney-Genitourinary System}

The kidney parenchyma is one of the most frequently involved sites in essentially all systemic amyloidoses including AL, AA, ALECT2, and several of the hereditary amyloidoses [21] (Fig. 1). While AL is still the most common form of amyloidosis in the developed world, the worldwide distribution of amyloidosis types depends on ethnicity and/or geographic location (see above). In a recent series of 474 patients with biopsy-proven renal amyloidosis from the Mayo Clinic, $86 \%$ were immunoglobulin derived, $7 \%$ were AA derived, $3 \%$ were ALECT2 derived, and $1 \%$ were fibrinogen A derived [21]. However, among Mexican Americans, ALECT2 accounts for $54 \%$ of renal amyloid diagnoses, being therefore even more prevalent than AL [22]. Similarly, among Egyptians, renal ALECT2 was the second (after AA) most common amyloidosis at $31 \%$ [19]. Thus, ALECT2 appears to represent an important and most probably under-recognized cause of chronic kidney disease among Mexican Americans, Egyptians, and other ethnic groups around the world [19, 22, 24, 25].

Renal amyloidoses most commonly involve the glomeruli and are typically associated with proteinuria, which, in turn, prompts a kidney biopsy. In routine sections (H\&E stained), amyloid is typically suspected through the presence of amorphous "hyaline" deposits, which are also weakly PAS positive and show loss of argyrophilia [21, 50, 75]. However, early deposits of amyloid may be inconspicuous and, therefore, a Congo red stain should be performed, not only to confirm the suspicion of amyloid but also to rule it out [50]. If early depos- its are missed, minimal change disease may be misdiagnosed. Typically, amyloid deposits are first seen in the mesangium and, subsequently, also involve the capillaries; ultimately, they may obliterate the entire glomerulus, mimicking sclerosis or diabetic nephropathy. While extraglomerular deposits of amyloid are typically associated with glomerular involvement, in some cases, deposits of amyloid may be purely extraglomerular and, clinically, may be associated with renal failure without insignificant proteinuria [75]. In ALECT2, cortical interstitium is typically involved, while glomeruli may be spared. In some patients with AA, AApoAI, or AApoAIV, amyloid deposits may be limited to the medullary interstitium. While in certain mutations in ATTR, glomeruli may be involved, typically, amyloid is limited to the deep medulla $[75,76]$.

Rarely, patients presenting with amyloid in the urinary tract are affected by localized amyloidosis. Small fiber neuropathy and endocrine involvement in systemic amyloidoses (AL, ATTR) may be associated with lower genitourinary dysfunction and/or functional impairment of the testes and adrenal glands [77].

\section{Heart}

The major systemic amyloidoses with clinically significant involvement of the heart include AL and ATTR, both wild type and hereditary; very rarely, other hereditary amyloidoses (AApoAI, AFib, ALys, and AGel) may also involve the heart $[7-9,28,29,31,78]$ (Fig. 1). Cardiac involvement in AA amyloidosis is rare and usually not clinically significant; vascular deposits of amyloid may also be seen in systemic $\mathrm{A} \beta 2 \mathrm{M}$ in dialysis patients [28].

Cardiac amyloidosis is an underdiagnosed cause of heart failure. Cardiovascular manifestations are rather nonspecific, with arrhythmias, conduction blocks, and congestive heart failure being typical. Nevertheless, ventricular wall thickening with preserved ejection fraction and absence of left ventricular dilatation is suspicious for amyloidosis and cardiac ATTR was reported in 13\% of patients with this condition [78]. Five percent of patients with presumed hypertrophic cardiomyopathy had a TTR gene mutation and $8 \%$ of patients with amyloidotic carpal tunnel syndrome also had cardiac ATTR [79]. Cardiac ATTR may also be associated with lumbar spinal stenosis $[43,46]$. By PYP scintigraphy, $16 \%$ of patients with aortic stenosis and up to $3 \%$ of all individuals older than $75 \mathrm{had}$ scans indicative of TTR cardiac amyloidosis [49]. Genetic testing is necessary to differentiate between ATTRv and ATTRwt cardiomyopathy. Moreover, among ATTRv, certain mutations are typically associated with cardiomyopathy. 
The true incidence of ATTRwt is difficult to determine but it is suspected of being widely underdiagnosed. Some patients may be relatively asymptomatic, while others, predominantly males, may develop congestive heart failure and arrhythmias with massive amyloid deposits.

In $\mathrm{AL}$, approximately $70 \%$ of patients have cardiac involvement, which confers a poor prognosis and warrants prompt specific treatment $[6,7]$. Compared to patients with ATTR, patients with cardiac involvement in AL have a greater hemodynamic impairment, despite a lesser amyloid load. Experimental data suggests that in AL, there is a direct cardiotoxic effect of amyloidogenic light chains, which plays a significant role in driving the cardiac function compromise [3].

Although the PYP scan has emerged as a helpful diagnostic modality in patients with cardiac ATTR (and no evidence of MGUS), definitive diagnosis requires an endomyocardial biopsy [41, 49, 78].

In $\mathrm{AL}$, amyloid deposits are seen diffusely infiltrating peri-myocyte connective tissue ("chicken-wire" pattern). In contrast, in ATTRwt, myocardial amyloid deposits are patchy in distribution; intramural coronary arteries may also be involved in both types $[50,80]$. Since early deposits of amyloid can be rather subtle on routine pathology, it is prudent to examine a Congo red stain of all native heart biopsies.

Light chain deposition disease, a systemic deposition of non-fibrillar deposits of immunoglobulin light chain that typically involves the kidney, may also involve the heart.

\section{Peripheral Nerves and Autonomic System}

Amyloidosis involving peripheral nerve includes $\mathrm{AL}$ and the hereditary amyloidoses, ATTRv, AGel, AApoAI, $A F i b$, and $A \beta 2 M v ; A A$ is not known to cause peripheral neuropathy $[28,81]$ (Fig. 1).

By definition, peripheral nerve amyloidosis includes infiltrative deposits of amyloid with associated clinical manifestations [81]. A diagnosis of amyloid peripheral neuropathy is often delayed because the clinical features may mimic many other neuropathies. Overall, amyloidosis is a relatively rare cause of peripheral neuropathy - in the Mayo Clinic peripheral nerve laboratory, amyloid peripheral neuropathy accounted for only $3 \%$ of cases [81-83].

Currently, fat aspiration is considered insensitive and nonspecific in the evaluation of amyloid neuropathy and, therefore, sural nerve biopsy is recommended, while in cases of focal amyloid (amyloidoma), a targeted biopsy is needed. The sensitivity of sural nerve biopsy in ATTRv is approximately $83 \%$ [53].

Pathology and Classification of Amyloidosis in Clinical Practice
Hereditary amyloidosis derived from transthyretin variant (ATTRv) is the most common amyloidosis associated with polyneuropathy in the US and also worldwide $[28,81]$. In AL, peripheral neuropathy is reported to occur in $17-35 \%$ of patients. The clinical presentation is dominated by acral extremity pain and autonomic features (hypohidrosis, gastrointestinal dysmotility, orthostasis, and impotence).

Amyloidosis derived from a gelsolin variant, AGelv, is much less common than ATTRv, and is associated with predominant seventh cranial nerve neuropathy as well as (later in the course of the disease) distal polyneuropathy. Neuropathy may occur in AApoAI and certain mutations in $\mathrm{AFib}$, and $\mathrm{A} \beta 2 \mathrm{Mv}$ has been reported with autonomic and subsequent symmetric sensorimotor axonal polyneuropathy $[28,40,81]$.

Amyloid mimickers include non-amyloidotic immunoglobulin deposition disease and diabetes.

\section{Gastrointestinal Tract}

Gastrointestinal tract is frequently involved in systemic amyloidoses - most commonly AL, followed by AA and hereditary types; ALECT2 involvement was also recently reported [22, 26, 64, 84] (Fig. 1). A localized form of amyloid - "amyloidoma" - can also occur. The clinical presentation of gastrointestinal amyloid may range from relatively vague signs and symptoms to frank diarrhea, malabsorption, hematochezia or hematemesis, obstipation, obstruction or pseudo-obstruction, and perforation. Gastric involvement may include nausea, vomiting, and epigastric pain or gastric outflow obstruction.

In general, the pattern of amyloid deposition is not reliable in amyloid typing. The earliest amyloid deposits involve small arterioles of the submucosa and later extend to involve the lamina propria, muscularis mucosa, submucosa, and muscularis propria; polypoid amyloid deposits have been reported in the stomach, small bowel, and colonic mucosa (Fig. 2a, b). Subserosal connective tissue deposits may extend into the adjacent mesentery. Colonic mucosal amyloid may mimic collagenous colitis. In ATTRwt, deposits of amyloid are typically limited to the vasculature [46].

Liver

The liver is frequently involved in systemic amyloidoses, including AL, ALECT2, AA, AApoAI, and ALys [5-7, 28,85 ] (Fig. 1). In a recent series of 130 patients with liver amyloidosis diagnosed by proteomics at the Mayo Clinic, $62 \%$ were AL, $25 \%$ were ALECT2, $7 \%$ were AApo AI, $4 \%$ were AA, $2 \%$ were ATTR, and $1 \%$ ALys.

Acta Haematol 2020;143:322-334

DOI: $10.1159 / 000506696$ 
Symptomatic dysfunction due to amyloid is uncommon and typically occurs late; hence, liver biopsy remains the gold standard for diagnosis. Patterns of hepatic amyloid involve sinusoidal, globular, arteriolar and/or capsular, and portal. Although transthyretin is primarily manufactured in the liver, amyloid deposits are limited to the vasculature and the capsule. ALECT2 produces amyloid with a globular pattern, which may be relatively specific for this amyloidosis type. However, similar to other organs, the pattern of amyloid deposition is not reliable in diagnosis of the amyloid type. Light chain deposition disease is a mimicker of hepatic amyloidosis; however, its deposits lack affinity for Congo red. In the liver, ALECT2 appears to be the second most commonly diagnosed amyloid type [85].

\section{Lungs}

Pulmonary amyloid deposits may be associated with a systemic disease or represent localized amyloidosis [48, 86]. Lung involvement in systemic amyloidosis is frequent, representing the fifth most common biopsy site yielding positivity for amyloid. The most common form of amyloidosis is AL (about 80\%) followed by ATTR (both hereditary and wild type), AA, AApoAIV, and $\mathrm{A} \beta 2 \mathrm{M}$ (Fig. 1). Localized pulmonary amyloidosis is relatively rare and most frequently of the AL type, associated with MALT lymphoma [48].

Light chain deposition disease and crystal-storing histiocytosis may form pulmonary deposits mimicking amyloid.

\section{Other Tissues}

Recently, several studies reported on positivity for ATTR amyloid in $10 \%$ of carpal tunnel and $33-44 \%$ of lumbar stenosis surgery specimens [43-46]. There is growing evidence that amyloid in these sites may be asso- ciated with an early cardiac amyloidosis, at least in some patients. It may also be prudent to look for other possible biopsies/pathology specimens from a given patient and, retrospectively, to test them for the presence of amyloid.

\section{Conclusions}

1 Early diagnosis of amyloidosis continues to pose a significant challenge and requires the participation of many clinical and laboratory specialties.

2 Determination of the protein type is imperative before specific therapy can be implemented.

3 While mass spectrometry has emerged as the preferred method of amyloid typing, careful application of immune methods is still clinically useful but caution and experience, as well as awareness of the limitations of each method, are necessary in their interpretation.

4 While the spectrum of amyloidoses continues to expand, it is critical to distinguish between those that are currently treatable versus those that are untreatable and to avoid causing harm by inappropriate treatment.

\section{Disclosure Statement}

The author declares no conflict of interest.

\section{Funding Sources}

None.

\section{Author Contribution}

Maria M. Picken, MD, $\mathrm{PhD}$ is the sole author of this paper.

\section{References}

1 Benson MD, Buxbaum JN, Eisenberg DS, Merlini G, Saraiva MJ, Sekijima Y, et al. Amyloid nomenclature 2018: recommendations by the International Society of Amyloidosis (ISA) nomenclature committee. Amyloid. 2018 Dec; 25(4):215-9.

2 Chiti F, Dobson CM. Protein Misfolding, Amyloid Formation, and Human Disease: A Summary of Progress Over the Last Decade. Annu Rev Biochem. 2017 Jun;86(1):27-68.

3 Nuvolone M, Merlini G. Systemic amyloidosis: novel therapies and role of biomarkers. Nephrol Dial Transplant. 2017 May;32(5): $770-80$.
4 Misra P, Blancas-Mejia LM, Ramirez-Alvarado M. Mechanistic Insights into the Early Events in the Aggregation of Immunoglobulin Light Chains. Biochemistry. 2019 Jul;58(29): 3155-68.

5 Wechalekar AD, Gillmore JD, Hawkins PN. Systemic amyloidosis. Lancet. 2016 Jun; 387(10038):2641-54.

6 Gertz MA. Immunoglobulin light chain amyloidosis: 2018 Update on diagnosis, prognosis, and treatment. Am J Hematol. 2018 Sep;93(9): 1169-80.
7 Merlini G, Dispenzieri A, Sanchorawala V, Schönland SO, Palladini G, Hawkins PN, et al. Systemic immunoglobulin light chain amyloidosis. Nat Rev Dis Primers. 2018 Oct;4(1):38.

8 Gertz MA, Mauermann ML, Grogan M, Coelho T. Advances in the treatment of hereditary transthyretin amyloidosis: A review. Brain Behav. 2019 Sep;9(9):e01371.

9 Maurer MS, Bokhari S, Damy T, Dorbala S, Drachman BM, Fontana M, et al. Expert Consensus Recommendations for the Suspicion and Diagnosis of Transthyretin Cardiac Amyloidosis. Circ Heart Fail. 2019 Sep;12(9): e006075. 
10 Adams D, Ando Y, Beirão JM, Coelho T, Gertz MA, Gillmore JD, et al. Expert consensus recommendations to improve diagnosis of ATTR amyloidosis with polyneuropathy [Epub ahead of print] [Review]. J Neurol. 2020. https://doi. org/10.1007/s00415-019-09688-0.

11 Picken MM. Alect2 amyloidosis: primum non nocere (first, do no harm). Kidney Int. 2014 Aug;86(2):229-32.

12 Naiki H, Sekijima Y, Ueda M et al.Human Amyloidosis, Still Intractable but Becoming Curable: The Essential Role of Pathological Diagnosis in the Selection of Type-Specific Therapeutics. Pathol Int. 2020 Jan 21 [Online ahead of print]. PMID: 31961039, DOI: https://doi. org/10.1111/pin.12902.

13 Quock TP, Yan T, Chang E, Guthrie S, Broder MS. Epidemiology of AL amyloidosis: a realworld study using US claims data. Blood Adv. 2018 May;2(10):1046-53.

14 Gertz MA, Comenzo R, Falk RH, Fermand JP, Hazenberg BP, Hawkins PN, et al. Definition of organ involvement and treatment response in immunoglobulin light chain amyloidosis (AL): a consensus opinion from the 10th International Symposium on Amyloid and Amyloidosis, Tours, France, 18-22 April 2004. Am J Hematol. 2005 Aug;79(4):319-28.

15 Ombrello AK, Aksentijevich IA. Amyloidosis. In: Amyloid and related disorders - surgical pathology and clinical correlations, 2nd ed. Humana Press 2015, Ed: Picken MM, Herrera GA, Dogan A. pp 31-53.

16 Westermark GT, Fändrich M, Westermark P. AA amyloidosis: pathogenesis and targeted therapy. Annu Rev Pathol. 2015;10(1):321-44.

17 Lane T, Pinney JH, Gilbertson JA, Hutt DF, Rowczenio DM, Mahmood S, et al. Changing epidemiology of AA amyloidosis: clinical observations over 25 years at a single national referral centre. Amyloid. 2017 Sep;24(3):162-6.

18 Papa R, Lachmann HJ. Secondary, AA, Amyloidosis. Rheum Dis Clin North Am. 2018 Nov; 44(4):585-603.

19 Larsen CP, Ismail W, Kurtin PJ, Vrana JA, Dasari S, Nasr SH. Leukocyte chemotactic factor 2 amyloidosis (ALECT2) is a common form of renal amyloidosis among Egyptians. Mod Pathol. 2016 Apr;29(4):416-20.

20 Blank N, Hegenbart U, Dietrich S, Brune M, Beimler J, Röcken C, et al. Obesity is a significant susceptibility factor for idiopathic AA amyloidosis. Amyloid. 2018 Mar;25(1):37-45.

21 Said SM, Sethi S, Valeri AM, Leung N, Cornell LD, Fidler ME, et al. Renal amyloidosis: origin and clinicopathologic correlations of 474 recent cases. Clin J Am Soc Nephrol. 2013 Sep; 8(9):1515-23.

22 Larsen CP, Beggs ML, Wilson JD, Lathrop SL. Prevalence and organ distribution of leukocyte chemotactic factor 2 amyloidosis (ALECT2) among decedents in New Mexico. Amyloid. 2016;23(2):119-23.

23 Benson MD, James S, Scott K, Liepnieks JJ, Kluve-Beckerman B. Leukocyte chemotactic factor 2: A novel renal amyloid protein. Kidney Int. 2008 Jul;74(2):218-22.
24 Hutton HL, DeMarco ML, Magil AB, Taylor P. Renal leukocyte chemotactic factor 2 (LECT2) amyloidosis in First Nations people in Northern British Columbia, Canada: a report of 4 cases. Am J Kidney Dis. 2014 Nov;64(5):790-2. 25 Rezk T, Gilbertson JA, Rowczenio D, Bass P, Lachmann HJ, Wechalekar AD, et al. Diagnosis, pathogenesis and outcome in leucocyte chemotactic factor 2 (ALECT2) amyloidosis. Nephrol Dial Transplant. 2018 Feb;33(2):241-7.

26 Said SM, Sethi S, Valeri AM, Chang A, Nast $\mathrm{CC}$, Krahl L, et al. Characterization and outcomes of renal leukocyte chemotactic factor 2-associated amyloidosis. Kidney Int. 2014 Aug;86(2):370-7.

27 Nasr SH, Dogan A, Larsen CP. Leukocyte cellderived chemotaxin 2-associated amyloidosis: a recently recognized disease with distinct clinicopathologic characteristics. Clin J Am Soc Nephrol. 2015 Nov; 10(11):2084-93.

28 Benson MD. The Hereditary Amyloidoses. In: Amyloid and related disorders - surgical pathology and clinical correlations, 2nd ed. Humana Press 2015, Ed: Picken MM, Herrera GA, Dogan A. pp 65-80. https://doi.org/10.1007/ 978-3-319-19294-9_5.

29 Maurer MS, Hanna M, Grogan M, Dispenzieri A, Witteles R, Drachman B, et al.; THAOS Investigators. Genotype and Phenotype of Transthyretin Cardiac Amyloidosis: THAOS (Transthyretin Amyloid Outcome Survey). J Am Coll Cardiol. 2016 Jul;68(2):161-72.

30 Parman Y, Adams D, Obici L, Galán L, Guergueltcheva V, Suhr OB, et al.; European Network for TTR-FAP (ATTReuNET). Sixty years of transthyretin familial amyloid polyneuropathy (TTR-FAP) in Europe: where are we now? A European network approach to defining the epidemiology and management patterns for TTR-FAP. Curr Opin Neurol. 2016 Feb;29 Suppl 1:S3-13.

31 Rubin J, Maurer MS. Cardiac Amyloidosis: Overlooked, Underappreciated, and Treatable. Annu Rev Med. 2020 Jan;71(1):203-19.

32 Gillmore JD, Lachmann HJ, Rowczenio D, Gilbertson JA, Zeng CH, Liu ZH, et al. Diagnosis, pathogenesis, treatment, and prognosis of hereditary fibrinogen $\mathrm{A}$ alpha-chain amyloidosis. J Am Soc Nephrol. 2009 Feb;20(2):444-51.

33 Stangou AJ, Banner NR, Hendry BM, Rela M, Portmann B, Wendon J, et al. Hereditary fibrinogen A $\alpha$-chain amyloidosis: phenotypic characterization of a systemic disease and the role of liver transplantation. Blood. 2010 Apr; 115(15):2998-3007.

34 Jacobson DR, Alexander AA, Tagoe C, Buxbaum JN. Prevalence of the amyloidogenic transthyretin (TTR) V122I allele in $14333 \mathrm{Af}-$ rican-Americans. Amyloid. 2015;22(3):171-4.

35 Suhr OB, Lundgren E, Westermark P. One mutation, two distinct disease variants: unravelling the impact of transthyretin amyloid fibril composition. J Intern Med. 2017 Apr;281(4): $337-47$.

36 Phull P, Sanchorawala V, Connors LH, Doros G, Ruberg FL, Berk JL, et al. Monoclonal gammopathy of undetermined significance in systemic transthyretin amyloidosis (ATTR). Amyloid. 2018 Mar;25(1):62-7.
37 Picken MM. Fibrinogen amyloidosis: the clot thickens! Blood. 2010 Apr;115(15):2985-6.

38 Tavares I, Moraira L, Costa PP, Lobato L. Fibrinogen A alpha chain amyloidosis: a nonnegligible cause of chronic kidney disease in dialysis patients. Amyloid. 2017;24(sup 1): 153-4. https://doi.org/10.1080/13506129.2017 .1281119 .

39 Chapman J, Dogan A. Fibrinogen alpha amyloidosis: insights from proteomics. Expert Rev Proteomics. 2019 Sep;16(9):783-93.

40 Valleix S, Drunat S, Philit JB, Adoue D, Piette JC, Droz D, et al. Hereditary renal amyloidosis caused by a new variant lysozyme W64R in a French family. Kidney Int. 2002 Mar;61(3): 907-12.

41 Grogan M, Scott CG, Kyle RA, Zeldenrust SR, Gertz MA, Lin G, et al. Natural History of Wild-Type Transthyretin Cardiac Amyloidosis and Risk Stratification Using a Novel Staging System. J Am Coll Cardiol. 2016 Sep; 68(10):1014-20.

42 Brunjes DL, Castano A, Clemons A, Rubin J, Maurer MS. Transthyretin Cardiac Amyloidosis in Older Americans. J Card Fail. 2016 Dec; 22(12):996-1003.

43 Yanagisawa A, Ueda M, Sueyoshi T, Okada T, Fujimoto T, Ogi Y, et al. Amyloid deposits derived from transthyretin in the ligamentum flavum as related to lumbar spinal canal stenosis. Mod Pathol. 2015 Feb;28(2):201-7.

44 Aus dem Siepen F, Hein S, Prestel S, Baumgärtner C, Schönland S, Hegenbart U, et al. Carpal tunnel syndrome and spinal canal stenosis: harbingers of transthyretin amyloid cardiomyopathy? Clin Res Cardiol. 2019 Dec;108(12): 1324-30.

45 Sperry BW, Reyes BA, Ikram A, Donnelly JP, Phelan D, Jaber WA, et al. Tenosynovial and Cardiac Amyloidosis in Patients Undergoing Carpal Tunnel Release. J Am Coll Cardiol. 2018 Oct;72(17):2040-50.

46 Westermark P. Localized amyloidoses and amyloidoses associated with aging outside the central nervous system. In: Amyloid and related disorders - surgical pathology and clinical correlations, 2nd ed. Humana Press 2015, Ed: Picken MM, Herrera GA, Dogan A. pp 95-120. https://doi.org/10.1007/978-3-319-19294-9_7.

47 Fayyaz AU, Melanie C, Bois MC, Dasari S, et al. Amyloidosis in surgically resected atrial appendages: a study of 345 consecutive cases with clinical implications. Mod Pathol. 2019 Nov 13 [EPub ahead of print]. PMID 31723241, https://doi.org/10.1038/s41379-019-0407-5.

48 Grogg KL, Aubry MC, Vrana JA, Theis JD, Dogan A. Nodular pulmonary amyloidosis is characterized by localized immunoglobulin deposition and is frequently associated with an indolent B-cell lymphoproliferative disorder. Am J Surg Pathol. 2013 Mar;37(3):406-12.

49 Flaherty KR, Morgenstern R, Pozniakoff T, DeLuca A, Castano A, Maurer MS, et al. 99mTechnetium pyrophosphate scintigraphy with cadmium zinc telluride cameras is a highly sensitive and specific imaging modality to diagnose transthyretin cardiac amyloidosis. J Nucl Cardiol. 2019 Aug: https://doi.org/10.1007/ s12350-019-01831-8.; Epub ahead of print. 
50 Picken MM. Modern approaches to the treatment of amyloidosis: the critical importance of early detection in surgical pathology. Adv Anat Pathol. 2013 Nov;20(6):424-39.

51 Javidiparsijani S, Picken MM. Should the Reporting of Bone Marrow Positivity for Amyloid Be Revised? A Critical Assessment Based On 66 Biopsies From a Single Institution [Online ahead of print]. Arch Pathol Lab Med. 2020 Jan;arpa.2019-0324-OA.

52 Picken MM, Herrera GA. Thioflavin T stain: an easier and more sensitive method for amyloid detection. In: Amyloid and related disorders surgical pathology and clinical correlations, 2nd ed. Humana Press 2015, Ed: Picken MM, Herrera GA, Dogan A. pp 225-227. https://doi. org/10.1007/978-3-319-19294-9_15.

53 Fine NM, Arruda-Olson AM, Dispenzieri A, Zeldenrust SR, Gertz MA, Kyle RA, et al. Yield of noncardiac biopsy for the diagnosis of transthyretin cardiac amyloidosis. Am J Cardiol. 2014 May;113(10):1723-7.

54 Quarta CC, Gonzalez-Lopez E, Gilbertson JA, Botcher N, Rowczenio D, Petrie A, et al. Diagnostic sensitivity of abdominal fat aspiration in cardiac amyloidosis. Eur Heart J. 2017 Jun; 38(24):1905-8.

55 van Gameren II, Hazenberg BP, Bijzet J, van Rijswijk MH. Diagnostic accuracy of subcutaneous abdominal fat tissue aspiration for detecting systemic amyloidosis and its utility in clinical practice. Arthritis Rheum. 2006 Jun; 54(6):2015-21.

56 Garcia Y, Collins AB, Stone JR. Abdominal fat pad excisional biopsy for the diagnosis and typing of systemic amyloidosis. Hum Pathol. 2018 Feb;72:71-9.

57 Vrana JA, Theis JD, Dasari S, Mereuta OM, Dispenzieri A, Zeldenrust SR, et al. Clinical diagnosis and typing of systemic amyloidosis in subcutaneous fat aspirates by mass spectrometry-based proteomics. Haematologica. 2014 Jul;99(7):1239-47.

58 Lee JC, Connors LH, O’Hara CJ. Bone Marrow Biopsy and Its Utility in the Diagnosis of AL Amyloidosis. In: Picken MM, Herrera GA, Dogan A. Amyloid and Related Disorders, Surgical Pathology and Clinical Correlations. Humana Press; 2015, pp 343-353. https://doi. org/10.1007/978-3-319-19294-9_26.

59 Hasserjian RP, Goodman HJ, Lachmann HJ, Muzikansky A, Hawkins PN. Bone marrow findings correlate with clinical outcome in systemic AL amyloidosis patients. Histopathology. 2007 Apr;50(5):567-73.

60 Sungur C, Sungur A, Ruacan S, Arik N, Yasavul U, Turgan C, et al. Diagnostic value of bone marrow biopsy in patients with renal disease secondary to familial Mediterranean fever. Kidney Int. 1993 Oct;44(4):834-6.

61 Muchtar E, Dispenzieri A, Lacy MQ, Buadi FK, Kapoor P, Hayman SR, et al. Overuse of organ biopsies in immunoglobulin light chain amyloidosis (AL): the consequence of failure of early recognition. Ann Med. 2017 Nov;49(7):545-51.
62 Picken MM. Options for Amyloid Typing in Renal Pathology: The Advantages of Frozen Section Immunofluorescence and a Summary of General Recommendations Regarding Immunohistochemistry Methods. In: Picken, et al., editors. Amyloid and Related Disorders. 2nd ed. 2015. pp. 283-93.

63 Linke RP, Meinel A, Chalcroft JP. Sensitive and Reliable Immunohistochemical Typing of 21 Different Amyloid Classes in 782 Patients Using Amyloid-Type Specific Antibodies Directed Against the Amyloidotic Conformation. With Comments on the Gold Standard Debate. Amyloid. 2017;24(sup1):157-8.

64 Freudenthaler S, Hegenbart U, Schönland S, Behrens HM, Krüger S, Röcken C. Amyloid in biopsies of the gastrointestinal tract-a retrospective observational study on 542 patients. Virchows Arch. 2016 May;468(5):569-77.

65 Dogan A. Amyloidosis: insights from Proteomics. Annu Rev Pathol. 2017 Jan;12(1): 277-304.

66 Lavatelli F, di Fonzo A, Palladini G, Merlini G. Systemic amyloidoses and proteomics: the state of the art. EuPA Open Proteom. 2016 Feb; 11:4-10.

67 Picken MM. Proteomics and mass spectrometry in the diagnosis of renal amyloidosis. Clin Kidney J. 2015 Dec;8(6):665-72.

68 Nasr SH, Fidler ME, Said SM. Paraffin Immunofluorescence: A Valuable Ancillary Technique in Renal Pathology. Kidney Int Rep. 2018 Jul;3(6):1260-6.

69 Messias NC, Walker PD, Larsen CP. Paraffin immunofluorescence in the renal pathology laboratory: more than a salvage technique. Mod Pathol. 2015 Jun;28(6):854-60.

70 Gonzalez Suarez ML, Zhang P, Nasr SH, Sathick IJ, Kittanamongkolchai W, Kurtin PJ, et al. The sensitivity and specificity of the routine kidney biopsy immunofluorescence panel are inferior to diagnosing renal immunoglobulinderived amyloidosis by mass spectrometry. Kidney Int. 2019 Oct;96(4):1005-9.

71 Gilbertson JA, Theis JD, Vrana JA, Lachmann $\mathrm{H}$, Wechalekar A, Whelan C, et al. A comparison of immunohistochemistry and mass spectrometry for determining the amyloid fibril protein from formalin-fixed biopsy tissue. J Clin Pathol. 2015 Apr;68(4):314-7.

72 Rezk T, Gilbertson JA, Mangione PP, Rowczenio D, Rendell NB, Canetti D, et al. The complementary role of histology and proteomics for diagnosis and typing of systemic amyloidosis. J Pathol Clin Res. 2019 Jul;5(3):145-53.

73 Abildgaard N, Rojek AM, Møller HE, Palstrøm NB, Nyvold CG, Rasmussen LM, et al. Immunoelectron microscopy and mass spectrometry for classification of amyloid deposits. Amyloid. 2020 Mar;27(1):59-66.

74 Owen-Casey MP, Sim R, Cook HT, Roufosse CA, Gillmore JD, Gilbertson JA, et al. Value of antibodies to free light chains in immunoperoxidase studies of renal biopsies. J Clin Pathol. 2014 Aug;67(8):661-6.
75 Sethi S, Theis JD. Pathology and diagnosis of renal non-AL amyloidosis. J Nephrol. 2018 Jun;31(3):343-50.

76 Lobato L, Rocha A. Transthyretin amyloidosis and the kidney. Clin J Am Soc Nephrol. 2012 Aug;7(8):1337-46.

77 Picken MM, Dogan A. Amyloidoses of the kidney, the lower urinary and genital tracts (male and female), and the breast. In: Amyloid and related disorders - surgical pathology and clinical correlations, 2nd ed. Humana Press 2015, Ed: Picken MM, Herrera GA, Dogan A. pp 369-389.

78 Damy T, Costes B, Hagège AA, Donal E, Eicher JC, Slama M, et al. Prevalence and clinical phenotype of hereditary transthyretin amyloid cardiomyopathy in patients with increased left ventricular wall thickness. Eur Heart J. 2016 Jun;37(23):1826-34.

79 Milandri A, Farioli A, Gagliardi C, Longhi S, Salvi F, Curti S, et al. Carpal tunnel syndrome in cardiac amyloidosis: implications for early diagnosis and prognostic role across the spectrum of aetiologies [Online ahead of print]. Eur J Heart Fail. 2020 Jan;2020(Jan):23.

80 Larsen BT, Mereuta OM, Dasari S, Fayyaz AU, Theis JD, Vrana JA, et al. Correlation of histomorphological pattern of cardiac amyloid deposition with amyloid type: a histological and proteomic analysis of 108 cases. Histopathology. 2016 Apr;68(5):648-56.

81 Loavenbruck AJ, Singer W, Mauermann ML, Sandroni P, B Dyck PJ, Gertz M, et al. Transthyretin amyloid neuropathy has earlier neural involvement but better prognosis than primary amyloid counterpart: an answer to the paradox? Ann Neurol. 2016 Sep;80(3):401-11.

82 Prada V, Massucco S, Venturi C et al. Diagnostic Value of Sural Nerve Biopsy: Retrospective Analysis of Clinical Cases from 1981 to 2017. Front Neurol. 2018;10, 22 November 2019. https://doi.org/https://doi.org/10.3389/ fneur.2019.01218.

83 Luigetti M, Di Paolantonio A, Bisogni G, Romano A, Conte A, Barbato F, et al. Sural nerve biopsy in peripheral neuropathies: 30 -year experience from a single center. Neurol Sci. 2020 Feb;41(2):341-6.

84 Said SM, Grogg KL, Smyrk TC. Gastric amyloidosis: clinicopathological correlations in 79 cases from a single institution. Hum Pathol. 2015 Apr;46(4):491-8.

85 Chandan VS, Shah SS, Lam-Himlin DM, Petris GD, Mereuta OM, Dogan A, et al. Globular hepatic amyloid is highly sensitive and specific for LECT2 amyloidosis. Am J Surg Pathol. 2015 Apr;39(4):558-64.

86 Baumgart JV, Stuhlmann-Laeisz C, Hegenbart U, Nattenmüller J, Schönland S, Krüger S, et al.; Systemic Pulmonary Amyloidosis-Impact on Diagnostics and Clinical Management. Local vs. systemic pulmonary amyloidosis-impact on diagnostics and clinical management. Virchows Arch. 2018 Nov;473(5):627-37. 